\title{
Métodos de destruição de restos de cultura do algodoeiro e sobrevivência do bicudo
}

\author{
Edenilson Batista Ribeiro(1), Maria Aparecida Castellani( ${ }^{(1)}$, Carlos Alberto Domingues da Silva(2), \\ Thiago Lima Melo(1), Gustavo dos Santos Silva(1), Willian Santos do Vale ${ }^{(1)}$ e Alexsandra Sousa Santos ${ }^{(1)}$
}

\begin{abstract}
(1)Universidade Estadual do Sudoeste da Bahia, Departamento de Fitotecnia e Zootecnia, Estrada do Bem Querer, Km 04, Caixa Postal 95, CEP 45031-900 Vitória da Conquista, BA, Brasil. E-mail: edenilson.eng.agronomo@gmail.com, castellani@uesb.edu.br, thiagolimelo@bol.com.br, gustavosans@agronomo.eng.br, willyan.vale@hotmail.com, sandra_re2009@yahoo.com.br (2)Embrapa Algodão, Rua Osvaldo Cruz, no 143, Centenário, Caixa Postal 174, CEP 58107-720 Campina Grande, PB, Brasil. E-mail: carlos.domingues-silva@embrapa.br
\end{abstract}

Resumo - O objetivo deste trabalho foi avaliar a eficiência de métodos de destruição dos restos de cultura do algodoeiro (Gossypium hirsutum) para a redução da população remanescente do bicudo-do-algodoeiro (Anthonomus grandis). O experimento foi conduzido em blocos ao acaso, com quatro repetições e cinco tratamentos: roçagem, com aplicação de 2,4-D e beta-ciflutrina; roçagem, com aplicação de 2,4-D e glifosato; roçagem e gradagem; roçagem, com aplicação de 2,4-D e beta-ciflutrina, além de gradagem; e testemunha (sem destruição). A quantidade de bicudos foi determinada após a destruição dos restos de cultura do algodão, por contagem dos insetos capturados nas armadilhas de feromônio e daqueles encontrados dentro dos carimãs, no interior das gaiolas e na área externa. O número médio de bicudos adultos, capturados nas armadilhas de feromônio no interior das gaiolas, variou de 0,71 a 1,35 indivíduos. O maior número de carimãs e de bicudos dos carimãs, dentro e fora das gaiolas, foi observado na testemunha. Já o menor número de insetos foi observado nos tratamentos com gradagem e roçagem e naqueles com roçagem e gradagem com aplicação de 2,4-D e beta-ciflutrina, que são eficientes na redução de carimãs e de adultos do bicudo. Todos os métodos avaliados reduzem a quantidade de bicudos vivos no interior de carimãs.

Termos para indexação: Anthonomus grandis, Gossypium hirsutum, agroquímicos, destruição mecânica, sobrevivência.

\section{Methods of cotton stalk destruction and survival of boll weevil}

\begin{abstract}
The objective of this work was to evaluate the effectiveness of methods for the destruction of cotton (Gossypium hirsutum) stalks on the remainder population of cotton boll weevil (Anthonomus grandis). The experiment was carried out in a randomized complete block design with four replicates and five treatments: mowing and application of 2,4-D and beta-cyfluthrin; mowing and application of 2,4-D and glyphosate; mowing and harrowing; mowing and application of 2,4-D and beta-cyfluthrin, besides harrowing; and a control without destruction. Boll weevil amount was determined after the destruction of cotton crop residues by counting the caught insects both in pheromone traps and within the dry bolls inside the cages and in the outer area. The average number of boll weevil adults captured in pheromone traps within the cages ranged from 0.71 to 1.35 individuals. The highest number of dry bolls and boll weevils from dry bolls, in and out of cages, was observed in the control. The lowest number of insects was observed in the treatments with harrowing and mowing and in those with mowing and harrowing with application of 2,4-D and beta-cyfluthrin, which are efficient in the reduction of dry bolls and boll weevil adults. All the evaluated methods reduce the amount of live boll weevils inside dry bolls.
\end{abstract}

Index terms: Anthonomus grandis, Gossypium hirsutum, agrochemicals, mechanical destruction, survival.

\section{Introdução}

A cultura do algodoeiro (Gossypium hirsutum L.) é atacada por diversas espécies de insetos e ácaros, o que exige dos agricultores a adoção de estratégias de controle para garantir produções economicamente viáveis. Dentre os insetos-praga, destaca-se o bicudo-do-algodoeiro, Anthonomus grandis Boheman (Coleoptera, Curculionidae), responsável por ocasionar severas perdas à produção (Ramalho, 1994; Silva \& Ramalho, 2013). Estima-se que os prejuízos econômicos, provocados pelo bicudo à cultura do algodoeiro em 2012, variaram de 70 a 130 dólares por hectare (Soria et al., 2013). 
Em condições ambientais ideais, a planta de algodão pode rebrotar após a colheita e produzir estruturas reprodutivas adequadas à alimentação e oviposição do bicudo pelo período de três a quatro semanas (Lemon et al., 2003; Lu et al., 2010). Portanto, a destruição dos restos dessa cultura é uma importante prática cultural para o manejo de populações remanescentes do bicudo e de outras pragas, como a broca-da-raiz [Eutinobothrus brasiliensis Hambleton (Coleoptera: Curculionidae)]; pulgão [Aphis gossypii Glover, 1877 (Hemiptera: Aphididae)]; mosca-branca [Bemisia tabaci Gennadius, 1889 (Hemiptera: Aleyrodidae)] e lagarta-rosada [Pectinophora gossypiella Saunders, 1844 (Lepidoptera: Gelechiidae)], que podem continuar o seu desenvolvimento nas soqueiras (Torres, 2008; Izeppi et al., 2011; Bianchini \& Borges, 2013; Silva et al., 2013). Essa prática é geralmente realizada de maneira mecânica, pela trituração dos tecidos vegetais, mas os recentes avanços nas práticas agrícolas de conservação de solos requerem métodos alternativos, como por exemplo, a aplicação de herbicidas (Yang et al., 2006).

Estudada na década de 1960, a estratégia química em que se utiliza a associação inseticida e herbicida de forma simultânea apresentou controle satisfatório do bicudo nas condições de Louisiana, US (Cleveland \& Smith, 1964). O herbicida pode promover a migração do bicudo para outras áreas (Showler, 2008), e o inseticida lambda-cialotrina favorece a redução do potencial reprodutivo da praga (Greenberg et al., 2004a, 2007, 2010).

No Brasil, embora a prática de destruição dos restos de cultura do algodoeiro ao final da safra seja obrigatória por lei, na maioria dos estados produtores (Almeida et al., 2008), poucos são os conhecimentos sobre essa prática de cultivo originados de pesquisa (Bianchini \& Borges, 2013). A maioria dos estudos trata dos efeitos desses métodos de destruição sobre os resíduos vegetais do algodoeiro (Silva et al., 1999; Showler et al., 2006a; Yang et al., 2006; Bianchini \& Borges, 2013), mas os fatores específicos relacionados à mortalidade do bicudo, após a colheita, não foram avaliados.

Adultos de bicudo são capazes de sobreviver nos campos de algodão, em restos de plantas e frutos de algodão caídos ao solo, mesmo após a colheita (Showler, 2003). Portanto, se as operações de destruição dos restos de cultura do algodão não forem bem-sucedidas, algumas "maçãs" infestadas pelo bicudo poderão permanecer intactas no solo, no período da entressafra e, assim, possibilitar a emergência e dispersão do inseto para áreas de refúgio.

O objetivo deste trabalho foi avaliar a eficiência de métodos de destruição dos restos de cultura do algodoeiro (Gossypium hirsutum) para a redução da população remanescente do bicudo-do-algodoeiro (Anthonomus grandis).

\section{Material e Métodos}

O experimento foi implantado entre 25 e 26 de agosto de 2014 e conduzido até o dia 21 de outubro de 2014, após a colheita de um cultivo comercial de algodão com a cultivar 'FM 910', localizado na Fazenda Tapera

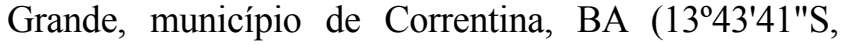
$45^{\circ} 46^{\prime} 36^{\prime \prime} \mathrm{W}$, a $849 \mathrm{~m}$ de altitude), em um talhão de 840 mil metros quadrados $(1.200 \times 700 \mathrm{~m})$ e área experimental de 113 mil metros quadrados (290x390 m), o que corresponde a $13,5 \%$ da área do talhão. As médias de temperatura e umidade relativa foram de $30,6^{\circ} \mathrm{C}$ e $24,5 \%$, respectivamente, durante o período de estudo. A colheita, nas parcelas, foi realizada com a máquina colhedeira John Deere 9970 (John Deere, Moline, IL, Estados Unidos), e a roçagem com a roçadeira Inroda (Inroda Máquinas Agrícolas e Implementos Agrícolas, Avaré, SP, Brasil). O equipamento foi regulado para corte das plantas a $20 \mathrm{~cm}$ de altura.

Utilizou-se o delineamento experimental de blocos ao acaso, com cinco tratamentos e quatro repetições. As parcelas apresentaram dimensões de $50 \times 54 \mathrm{~m}$, no total de $2.700 \mathrm{~m}^{2}$, distanciadas $30 \mathrm{~m}$ entre si. Os tratamentos utilizados foram os seguintes: roçagem e pulverizações com 2,4-D (1,21 kg de i.a. ha $\left.^{-1}\right)$ e beta-ciflutrina $\left(0,12 \mathrm{~kg}\right.$ de i.a. ha $\left.{ }^{-1}\right)$; roçagem e pulverizações com 2,4-D (1,21 kg de i.a. ha-1) e glifosato $(1,43 \mathrm{~kg}$ de i.a. ha-1 $)$; roçagem e gradagem; roçagem e pulverizações com 2,4-D $(1,21 \mathrm{~kg}$ de i.a. ha $\left.{ }^{-1}\right)$ e beta-ciflutrina $\left(0,12 \mathrm{~kg}\right.$ de i.a. ha $\left.{ }^{-1}\right)$, além de gradagem; e testemunha, sem destruição dos restos de cultura. Os herbicidas - 2,4-D e glifosato e o inseticida beta-ciflutrina foram selecionados para o estudo porque são normalmente utilizados pelos produtores de algodão do Oeste da Bahia.

As aplicações dos agrotóxicos foram executadas com o pulverizador Gladiador 2700 Stara, (Stara Indústria de Implementos Agrícolas, Não-Me-Toque, RS, Brasil), direcionado pelo sistema de posicionamento global, tendo-se utilizado o bico TXA8003VK do tipo 
cone, com vazão de $113 \mathrm{~L} \mathrm{ha}^{-1}$, utilizado à velocidade de $16 \mathrm{~km} \mathrm{~h}^{-1}$ e pressão de 5,0 bar. A gradagem foi realizada cinco dias após a aplicação dos agroquímicos, com auxílio de grade de disco, a $20 \mathrm{~cm}$ de profundidade e com duas passadas. Os agroquímicos foram aplicados ao final da noite, a $25^{\circ} \mathrm{C}$, com umidade relativa média de $42,5 \%$ e $3,5 \mathrm{~km} \mathrm{~h}^{-1}$ de velocidade do vento na direção leste-oeste.

Após a destruição dos restos de cultura do algodão, a quantidade de bicudos remanescentes foi determinada por meio da contagem dos insetos adultos capturados em armadilhas de feromônio e pela contagem dos bicudos presentes dentro das "maçãs" secas (carimãs), encontradas no interior das gaiolas e na área externa próxima às gaiolas. Foram utilizadas 20 gaiolas de policloreto de vinil $(32 \mathrm{~mm})$, com $2 \mathrm{~m}$ de altura $\mathrm{x}$ $2 \mathrm{~m}$ largura $\times 2 \mathrm{~m}$ comprimento, cobertas com tecido do tipo voil, que continha ao centro uma armadilha Accountrap de coloração verde fluorescente, com feromônio Luretape BW-10 (BioControle, Indaiatuba, SP, Brasil), para captura dos insetos.

As armadilhas foram observadas uma vez ao dia, na primeira semana, e a cada sete dias, nas cinco semanas subsequentes, por meio da contagem e coleta dos bicudos capturados. O número de bicudos contidos nos carimãs, coletados à superfície do solo, foi estimado após o início do experimento em dez áreas externas às gaiolas, aos 10 e 20 dias, e em 20 áreas, aos 38 dias. O tamanho de cada área amostrada foi de um metro quadrado. Os carimãs foram transportados ao Laboratório de Entomologia da Universidade Estadual do Sudoeste da Bahia (Uesb), onde foram dissecados, e os bicudos contidos em seu interior foram separados em vivos e mortos e contados.

Os dados foram submetidos ao teste de normalidade de Liliefors e transformados, quando necessário, para atender aos pré-requisitos da análise de variância. Os dados referentes ao número de bicudos capturados nas armadilhas de feromônio, ao de carimãs e ao de bicudos presentes nos carimãs foram submetidos à análise de variância, e as médias comparadas, pelo teste Tukey, a 5\% de probabilidade. Realizou-se a análise de correlação de Pearson (r) entre o número de insetos contidos nos carimãs e o número de carimãs.

\section{Resultados e Discussão}

O número médio de bicudos capturados nas armadilhas de feromônio, no interior das gaiolas, nos cinco tratamentos, variou entre de 0,71 a 1,35 indivíduos, tendo sido maior na testemunha, que apresentou média significativamente superior à dos tratamentos com roçagem, associada a pulverizações com 2,4-D e glifosato, e roçagem associada a pulverizações com 2,4-D e beta-ciflutrina mais gradagem (Figura 1). O número de bicudos capturados é consideravelmente menor do que aqueles encontrados por Showler (2008) em armadilhas de feromônio, instaladas em cultivos de algodão ao final da safra, em parcelas tratadas com mistura de tanque de inseticida e desfolhante, com médias superiores a 200 bicudos por armadilha, no ano de 2002, e acima de 20 bicudos por armadilha, em 2003, no tratamento controle após a colheita. A variação da quantidade de bicudos capturados pelo autor podem ser explicadas pelas diferenças entre os tamanhos das áreas disponibilizadas às armadilhas de feromônio para captura dos bicudos. No entanto, o baixo número de bicudos não deixa de ser relevante, quando se considera o elevado potencial biótico dessa praga. Um casal de bicudos pode produzir 250 mil descendentes, durante o ciclo de cultivo do algodão (Giesen, 2011); portanto, em altos níveis populacionais, as técnicas de

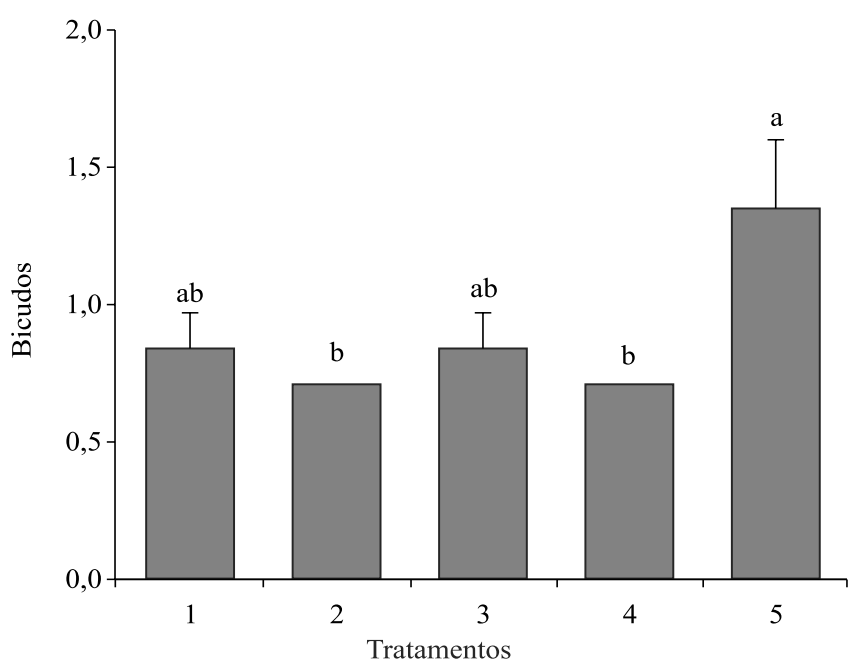

Figura 1. Número médio de adultos de bicudo-do-algodoeiro [Anthonomus grandis (Coleoptera: Curculionidae)], capturados em armadilhas de feromônio no interior de gaiolas, nos diferentes tratamentos. Tratamentos: 1, roçagem e aplicações de 2,4-D e beta-ciflutrina; 2, roçagem e aplicações de 2,4-D e glifosato; 3, roçagem e gradagem; 4, roçagem, gradagem e aplicações de 2,4-D e beta-ciflutrina; e 5, testemunha. Letras iguais sobre as barras não diferem pelo teste de Tukey, a 5\% de probabilidade. 
controle não são economicamente viáveis contra essa praga (Showler, 2003; El-Sayed et al., 2006).

O número de carimãs, observados dentro e fora das gaiolas nos cinco tratamentos, variou de 1,91 a 5,28 e de 147,50 a 672,42 , respectivamente. Observou-se maior número de carimãs na testemunha, com 5,28 dentro e 672,42 fora das gaiolas. O menor número de carimãs foi encontrado nos seguintes tratamentos: roçagem e gradagem, com 1,91 e 163,17 e roçagem e gradagem associadas a pulverizações com 2,4-D e beta-ciflutrina, com 1,98 e 147,50, dentro e fora das gaiolas, respectivamente (Figura 2). O método mecânico de roçagem associado à gradagem foi o mais
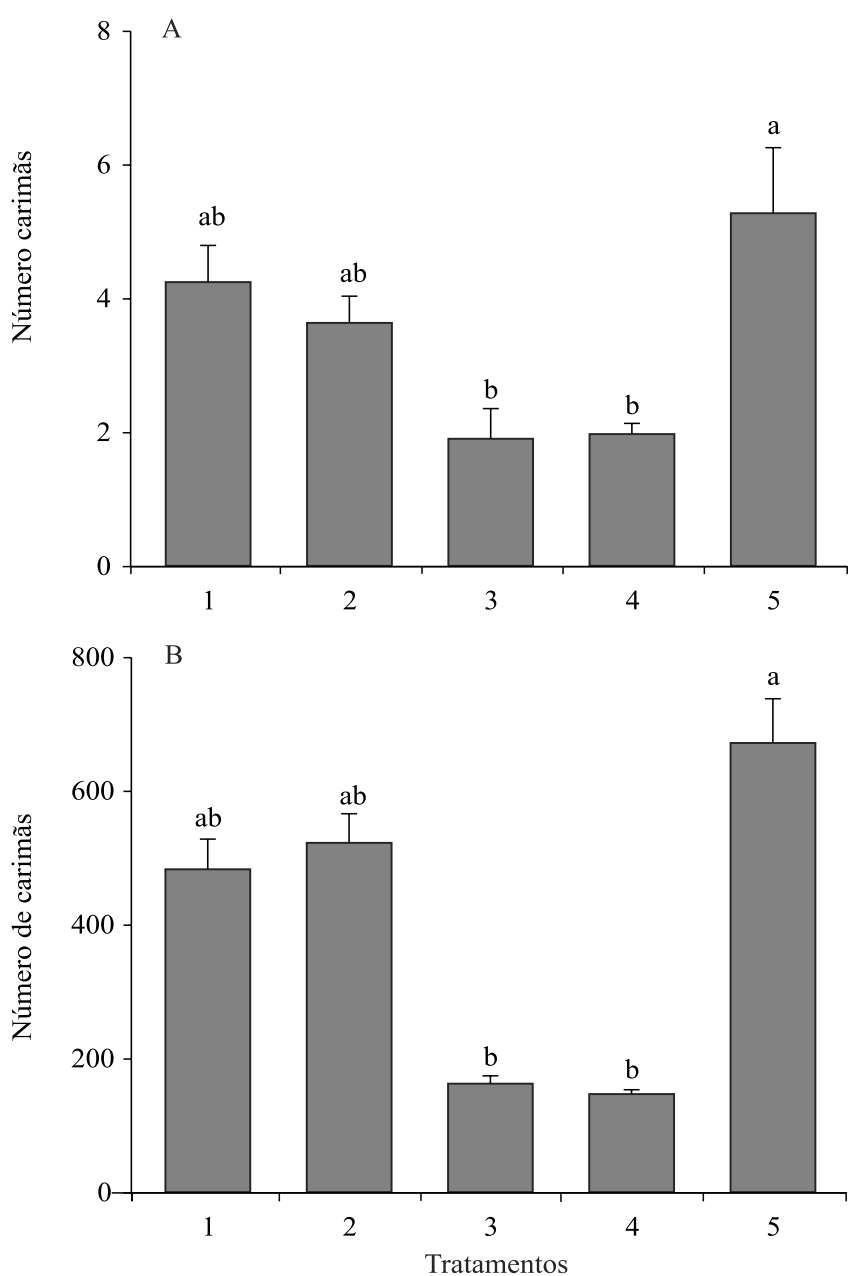

Figura 2. Número médio de carimãs, amostrados dentro (A) e fora (B) de gaiolas, nos diferentes tratamentos. Tratamentos: 1, roçagem e aplicações de 2,4-D e beta-ciflutrina; 2 roçagem e aplicações de 2,4-D e glifosato; 3, roçagem e gradagem; 4, roçagem, gradagem e aplicação de 2,4-D e beta-ciflutrina; e 5, testemunha. Letras iguais sobre as barras não diferem entre si, pelo teste de Tukey, a 5\% de probabilidade. eficiente para destruir os carimãs do algodoeiro. Isto é importante porque os bicudos podem sobreviver de uma safra a outra, abrigados nessas estruturas vegetais (Showler, 2006b, 2008). Além disso, o número de carimãs amostrados em todos os tratamentos se correlacionou positivamente ao número de bicudos observados no interior desses carimãs $(r=0,88, p=0,01)$. Com esses resultados, confirmou-se a importância da destruição dos restos de cultura do algodoeiro, em especial os carimãs, para reduzir a população de bicudos que poderiam colonizar as lavouras de algodão na safra seguinte (Pedigo \& Rice, 2009).

$\mathrm{O}$ número de bicudos coletados de carimãs dentro e fora das gaiolas, nos cinco tratamentos, variou de zero a dois exemplares e de zero a 63,8 exemplares, respectivamente. No interior das gaiolas, não houve diferença entre os tratamentos quanto ao número de bicudos coletados dos carimãs. No entanto, o número de bicudos coletados de carimãs, na parte externa das gaiolas, foi menor nos tratamentos com roçagem associada à gradagem e com roçagem associada à gradagem e pulverizações com 2,4-D e beta-ciflutrina (Figura 3). A prática da roçagem, seguida pela gradagem, apresenta uma eficiência de arranquio e redução de rebrota de 95,5 e $2,7 \%$, respectivamente (Silva et al., 1999). No presente trabalho, a operação da gradagem foi determinante para a redução de carimãs, provavelmente em razão da ação mecânica dos discos da grade, que cortam e incorporam ao solo os resíduos vegetais do algodoeiro. Em uma lavoura de algodão, no município de Jaboticabal, Estado de São Paulo, as práticas culturais de destruição dos restos de cultura por roçadeira e incorporação com gradagem, associada à pulverização da soqueira-isca, se mostraram mais eficientes na redução populacional do bicudo (Soares et al., 1994).

Com exceção dos bicudos coletados de carimãs da testemunha, na parte externa das gaiolas, todos os bicudos coletados dessas estruturas vegetais nos demais tratamentos encontravam-se mortos (Figura 3), o que mostra que os métodos de destruição dos restos culturais, avaliados no presente trabalho, contribuíram para as elevadas taxas de mortalidade da praga. O preparo do solo modifica os habitats onde algumas espécies de insetos-praga e insetos benéficos sobrevivem durante, pelo menos, parte de seu ciclo de vida (Greenberg et al., 2004b). Assim, modificações destes habitats podem alterar a sobrevivência e desenvolvimento dos 
insetos no solo e nas estrtuturas vegetais pertencentes ao dossel das plantas (Greenberg et al., 2012). Outro fator que pode aumentar a mortalidade dos bicudos, no interior das estruturas reprodutivas do algodoeiro, é a dessecação de larvas, pupas e adultos resultante das condições edafoclimáticas adversas (Cardoso et al., 2009). Assim, a temperatura alta e a umidade relativa baixa, registradas durante o período experimental, podem ter contribuído para aumentar a mortalidade, por dessecação, dos bicudos abrigados no interior
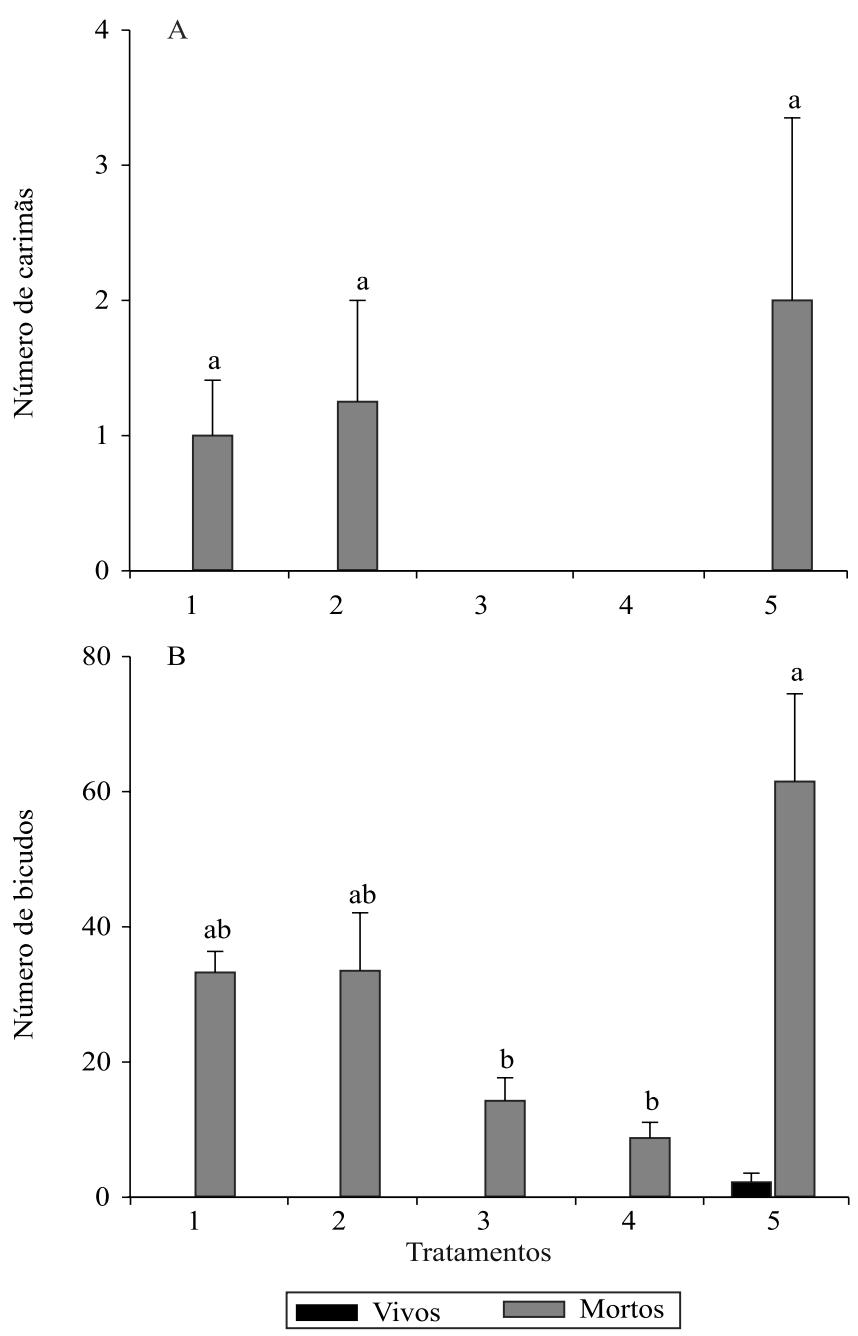

Figura 3. Número médio de adultos vivos e mortos do bicudo-do-algodoeiro [Anthonomus grandis (Coleoptera: Curculionidae)], coletados de carimãs dentro (A) e fora (B) das gaiolas, nos diferentes tratamentos. Tratamentos: 1 roçagem e aplicações de 2,4-D e beta-ciflutrina; 2, roçagem e aplicações de 2,4-D e glifosato; 3, roçagem e gradagem; 4, roçagem, gradagem e aplicação de 2,4-D e beta-ciflutrina; e 5, testemunha. Letras iguais sobre as barras não diferem entre si, pelo teste de Tukey, a 5\% de probabilidade. dos carimãs, especialmente naqueles tratamentos em que os carimãs foram danificados pela ação mecânica dos implementos agrícolas, utilizados na roçagem e gradagem.

Os resultados deste trabalho confirmam que os métodos de destruição da soqueira estudados podem reduzir as populações de bicudos sobreviventes no período de pós-colheita do algodoeiro e, assim, impedir e retardar sua infestação nos cultivos de algodão da próxima safra.

\section{Conclusões}

1. A roçagem associada à gradagem e a roçagem associada à gradagem com aplicações do herbicida 2,4-D e do inseticida beta-ciflutrina são métodos eficientes de destruição de restos culturais do algodão, quanto à redução de carimãs e de adultos do bicudo.

2. Os métodos de destruição de restos de cultura do algodão avaliados reduzem a quantidade de bicudos vivos no interior de carimãs.

\section{Agradecimentos}

À Fundação de Apoio à Pesquisa no Estado da Bahia, por concessão de bolsa.

\section{Referências}

ALMEIDA, R.P.; SILVA, C.A.D.; RAMALHO, F.S. Manejo integrado de pragas do algodão. In: BELTRÃO, N.E. de M.; AZEVEDO, D.M.P. de. (Ed.). O agronegócio do algodão no Brasil. 2.ed. Brasília: Embrapa Informação Tecnológica; Campina Grande: Embrapa Algodão, 2008. v.2, p.1034-1098.

BIANCHINI, A.; BORGES, P.H. de M. Evaluation of cotton stalks destroyers. Engenharia Agrícola, v.34, p.965-975, 2013. DOI: 10.1590/S0100-69162013000500008.

CARDOSO, U.P.; CASTELLANI, M.A.; FORTI, L.C.; MENEZES JÚNIOR, A. de O.; MOREIRA, A.A.; COLMENAREZ, Y.C.; OLIVEIRA, M.E.C.R. de; BITTENCOURT, M.A.L. Índice de infestação e fatores de mortalidade do bicudo-do-algodoeiro (Coleoptera: Curculionidae) no semi-árido do sudoeste da Bahia. Entomotropica, v.24, p.111-122, 2009.

CLEVELAND, T.C.; SMITH, G.L. Effects of postseason applications of insecticides, defoliants, and desiccants on diapausing boll weevils. Journal of Economic Entomology, v.57, p.527-529, 1964. DOI: 10.1093/jee/57.4.527.

EL-SAYED, A.M.; SUCKLING, D.M.; WEARING, C.H.; BYERS, J.A. Potential of mass trapping for long-term pest management and eradication of invasive species. Journal of Economic Entomology, v.99, p.1550-1554, 2006. DOI: 10.1093/ jee/99.5.1550. 
GIESEN, J.C. Boll weevil blues: cotton, myth, and power in the American South. Chicago: University of Chicago, 2011. 240p.

GREENBERG, S.M.; BRADFORD, J.M.; ADAMCZYK, J.J.; SMART, J.R.; LIU, T.-X. Insect population trends in different tillage systems of cotton in South Texas. Subtropical Plant Science, v.62, p.1-17, 2010 .

GREENBERG, S.M.; SHOWLER, A.T.; SAPPINGTON, T.W.; BRADFORD, J.M. Effects of burial and soil condition on postharvest mortality of boll weevils (Coleoptera: Curculionidae) in fallen cotton fruit. Journal of Economic Entomology, v.97, p.409-413, 2004b. DOI: 10.1093/jee/97.2.409.

GREENBERG, S.M.; ADAMCZYK, J.J.; ARMSTRONG, J.S. Principles and practices of integrated pest management on cotton in the lower Rio Grande Valley of Texas. In: LARRAMENDY, M.L.; SOLONESKI, S. (Ed.). Integrated pest management and pest control: current and future tactics. Rijeka: InTech, 2012. DOI: $10.5772 / 31467$

GREENBERG, S.M.; SAPPINGTON, T.W.; ELZEN, G.W.; NORMAN, J.W.; SPARKS, A.N. Effects of insecticides and defoliants applied alone and in combination for control of overwintering boll weevil (Anthonomus grandis; Coleoptera: Curculionidae) - laboratory and field studies. Pest Management Science, v.60, p.849-858, 2004a. DOI: 10.1002/ps.878.

GREENBERG, S.M.; SPARKS JR., A.N.; NORMAN JR., J.W.; COLEMAN, R.; BRADFORD, J.M.; YANG, C.; SAPPINGTON, T.W.; SHOWLER, A. Chemical cotton stalk destruction for maintenance of host-free periods for the control of overwintering boll weevil in tropical and subtropical climates. Pest Management Science, v.63, p.372-380, 2007. DOI: 10.1002/ps.1348.

IZEPPI, T.S.; GRIGOLLI, J.F.J.; SOUZA, L.A.; CROSARIOL NETTO, J.; BUSOLI, A.C. Ocorrência de Aphis gossypii (Hemiptera: Aphididae) e Cycloneda sanguinea (Coleoptera: Coccinelidae) em rebrota de plantas roçadas de cultivares comerciais de algodoeiro. Biológico, v.73, p.314-317, 2011.

LEMON, R.; STICHLER, C.; NORMAN JR., J. Cotton stalk destruction with herbicides. College Station: Texas A\&M University, 2003. SCS-2003-10.

LU, P.; DAVIS, R.F.; KEMERAIT, R.C. Effect of mowing cotton stalks and preventing plant re-growth on post-harvest reproduction of Meloidogyne incognita. Journal of Nematology, v.42, p.96-100, 2010 .

PEDIGO, L.P.; RICE, M.E. Entomology and pest management. $6^{\text {th }}$ ed. Upper Saddle River: Prentice Hall, 2009. 816p.

RAMALHO, F.S. Cotton pest management: part 4. A Brazilian perspective. Annual Review of Entomology, v.39, p.563-578, 1994. DOI: 10.1146/annurev.en.39.010194.003023.
SHOWLER, A.T.; FUNK, P.; ARMIJO, C. Effect of thermal defoliation on cotton leaf desiccation, senescence, post-harvest regrowth, and lint quality. Journal of Cotton Science, v.10, p.39-45, 2006a.

SHOWLER, A.T. Effects of routine late-season field operations on numbers of boll weevils (Coleoptera: Curculionidae) captured in large-capacity pheromone traps. Journal of Economic Entomology, v.96, p.680-689, 2003. DOI: 10.1093/jee/96.3.680.

SHOWLER, A.T. Efficiency of tank-mixing insecticide with defoliant against adult boll weevil (Coleoptera: Curculionidae) populations as determined by late-season field disturbance trapping. Subtropical Plant Science, v.60, p.58-65, 2008.

SHOWLER, A.T. Short-range dispersal and overwintering habitats of boll weevils (Coleoptera: Curculionidae) during and after harvest in the subtropics. Journal of Economic Entomology, v.99, p.1152-1160, 2006b. DOI: 10.1093/jee/99.4.1152.

SILVA, C.A.D.; RAMALHO, F.S. Kaolin spraying protects cotton plants against damages by boll weevil Anthonomus grandis Boheman (Coleoptera: Curculionidae). Journal of Pest Science, v.86, p.563-569, 2013. DOI: 10.1007/s10340-013-0483-0.

SILVA, C.A.D.; RAMALHO, F.S.; MIRANDA, J.E.; ALMEIDA, R.P.; RODRIGUES, S.M.M.; ALBUQUERQUE, F.A. Recomendações técnicas para o manejo integrado de pragas do algodoeiro no Brasil. Campina Grande: Embrapa Algodão, 2013. 12p. (Embrapa Algodão. Circular técnica, 135).

SILVA, O.R.R.F. da; CARVALHO, O.S.; VASCONCELOS, O.L.; SOARES, J.J.; QUEIROZ, J.C.; PAULA, D.F. de. Máquinas agrícolas: avaliação de diferentes métodos de destruição de restos culturais do algodoeiro. Revista Brasileira de Engenharia Agrícola e Ambiental, v.3, p.391-394, 1999.

SOARES, J.J.; BUSOLI, A.C.; YAMAMOTO, P.T.; BRAGA SOBRINHO, R. Efeito de práticas culturais de pós-colheita sobre populações do bicudo-do-algodoeiro, Anthonomus grandis Boheman, 1843. Pesquisa Agropecuária Brasileira, v.29, p.375-379, 1994.

SORIA, M.F.; THOMAZONI, D.; TACHINARDI, R.; DEGRANDE, P.E. Alerta para o bicudo-do-algodoeiro: breve panorama pré-safra 2012/13 e ações para o combate da praga. Cuiabá: Instituto Mato-grossense do Algodão, 2013. 4p. (IMAmt. Circular técnica, 3).

TORRES, J.B. Controle de pragas do algodoeiro: expectativas de mudanças. Ciência Agrícola, v.8, p.37-49, 2008.

YANG, C.; GREENBERG, S.M.; EVERITT, J.H.; NORMAN JR., J.W. Assessing cotton stalk destruction with herbicides using remote sensing technology. The Journal of Cotton Science, v.10, p.136-145, 2006.

Recebido em 24 de abril de 2015 e aprovado em 2 de setembro de 2015

Pesq. agropec. bras., Brasília, v.50, n.11, p.993-998, nov. 2015

DOI: 10.1590/S0100-204X2015001100001 